\title{
Towards analysis of the heritability of acquired characters: Transmutation and preinduction in
Daphnia.
}

\author{
Richard Woltereck*
}

maximilian@alumni.reed.edu

September 28, 2020

\begin{abstract}
A translation of Beitrag zur Analyse der Vererbung erworbener Eigenschaften: Transmutation und Präinduktion bei Daphniden, by Richard Woltereck. Published originally in the Verhandlungen der Deutschen zoologischen Gesellschaft in 1911.

In this paper, Woltereck presents some long-term culture experiments in which he attempts to induce heritable phenotypic changes (and thus implicitly genotypic changes) through unaccustomed environmental exposure. He does not explicitly select any particular phenotype from these cultures.

He finds that it is possible to induce changes in the first generation (which he calls "induction") and the second generation ("preinduction") after environmental stimuli are withdrawn, but no further.

He infers that these changes are due to effects on developing embryos for induction, and on germ cells within developing embryos for preinduction.

However, he concludes that he has not found evidence for the inheritance of acquired characters, as both preinduction and induction appear to be part of the animal's inherited reaction norm, and their effects are transient. He suggests a more inclusive definition of the reaction norm (and of inheritance) to accommodate this.

To explain how this might proceed, he draws upon the relatively new sciences of enzymology and immunology. He suggests that the idea of catalysis might be a good way of thinking about how genes can affect the reactions he is interested in, in that genes might be like enzymes (this is approximately three decades before Beadle and Tatum). Next, he suggests that we might understand the catalytic mechanism of such genes with an analogy to the inhibitory effects of antibodies. In short, he presents a model for gene-regulatory control of development (this is approximately five decades before Jacob and Monod).

Woltereck's speculations were not influential; various authors proposed enzyme theories of genes around the same time. Woltereck himself draws a hard and rather awkwardly over-emphasized line between the experimental results he presents one the one hand and his hypothetical model of inheritance on the other.
\end{abstract}

\section{TRANSLATOR'S NOTE}

*translation by Maximilian Oliver Press 2020

Throughout, I have included footnotes where appropriate; these are in italic and prefixed with MOP to distinguish them from Woltereck's notes. 


\section{Contents}

\section{Introduction}

1. Attempts towards a transmutation of helm height through longstanding action of altered environmental conditions.

2. Preinduction of helm height through environmental influence within a generation.

3. Attempt towards a transmutation of sexuality through long exposure to altered environmental conditions.

4. Preinduction of sex through influence of the environment during the sensible period within a generation.

5. How do preinduction and induction proceed?

6. Behavior of preinduction towards transmutation. Towards further analysis of the meaning of "reaction norm".

\section{INTRODUCTION}

There are two paths forward for analytical genetics, "pure line breeding as well as hybridization after Mendel's model'1 I have used the first to approach the problems of variation and heredity of quantitatively measurable traits. In these studies, I cannot avoid the problem of socalled inheritance of acquired (or compelled) characters.

Here I reference a previous paper $\left.\right|^{2}$ that I presented before you in 1909 regarding the analysis of some quantitative characters and their inheritance behavior, in which I dealt with some

\footnotetext{
${ }^{1}$ Johannsen, A genotype conception of heredity. American Naturalist 1911.

${ }^{2}$ These proceedings, 1909: Weitere experimentelle Untersuchungen über Artveränderung, speziell über das Wesen quantitativer Artunterschiede bei Daphniden. MOP note: For English, see here: https://osf.io/fvme6/
}

preliminary results in this direction. From a series of experiments it appeared that sustained action of the environment-when they affect the entire organism, including germ cells-led to an alteration of the "reaction norm", and thereby the inherited characters.

All variable characters, for example helm height (Fig. 1-2), have a reaction norm specific to and inherited by each elementary type. These characters express themselves accordingly.

From there, we must proceed under the assumption that all observable characters of an organism assume relative rather than absolute values, whether quantitative or qualitative in their variation. In other words, characters are the reactions of a substrate to inner and outer stimuli or causes. Substrate and stimulus together yield the observable state of a character.

As substrates, we mean the plasmasubstance of those groups of cells that develop the features of the character. Thus the Daphnia mantle cells are the substrate of helm development, the wing scale progenitors of Vanessa butterflies are the substrate of wing pigmentation, and so on.

We know the inner causes working upon the substrate as anlagen ("genes", "determinants" ${ }^{3}$ We have lately learned about these from Mendel's discoveries in hybridization, recently rediscovered and further clarified. We know now that these "genes" represent specific and non-decomposable units. However, today they are not an empty definition or hypothetical heritable units, but rather material realities of well-known (though still relative) efficacy and quantitative effect.

Finally, we know a third category of factors, which we bring together under the title "influences of the environment", which participate in the reactions that yield characters. Under this heading, we include the external effects of temperature, nourishment, chemical substances, hydration, soil conditions, and so on.

All three together-substrate plus gene plus environment-yield many reactions. More pre-

\footnotetext{
${ }^{3} \mathrm{MOP}$ note: I chose the German term because it is sometimes used in English, and he helpfully supplied clarifying terms.
} 
cisely, one and the same substrate (for example wing pigmentation) is capable of manifold reaction results (observable characters) under different grades of environment. This can be due to the action of one gene or multiple different genes.

The sum of all reactions affecting a character is inherited as a whole, with defined quantities for each stimulus and process in each type. This whole is the above-mentioned reaction norm of the relevant character substrate; from a different point of view, it is the reaction norm of the relevant variable character.

A heritable alteration of such characters, and thereby the type, appears only if the reaction norm is altered at any point. I believe that this event is best expressed with the old term "transmutation", which is not attached to any particular theory (the shorter term "mutation" is unfortunately thus afflicted), allowing us to focus on the heritable change in reaction without saying anything about the hypothetical "how".

The question which I posed to myself concerned whether such transmutations can be achieved through long exposure to a specific environmental influence. This question will concern us in the first and third parts of this article, for two types of variable characters.

While the further course of my experiments sharpens this question into quite another, it remains inexorably bound to the first. This further question regards whether, in this inheritance of reaction norms, variants of a character acquired through influence of the environment in one generation can be inherited in the next. And if this typical "inheritance of acquired characters" appears, can that occur without altering the heritable representation of that character (the genes)?

This question shall be discussed in the second and fourth sections. I will conclude with an attempt to penetrate deeper into the space between substrate, anlage, and environment under the prevailing reaction behaviors, in order to more precisely analyze the "reaction norm" which subsumes all of these factors.

\section{ATTEMPTS TOWARDS A} TRANSMUTATION OF HELM-HEIGHT THROUGH LONGSTANDING ACTION OF ALTERED ENVIRONMENTAL CONDITIONS.

\section{7-1909}

The principal results of my early experiments were that after some two years action of overfeeding and elevated temperature, a series of changes occurred in a D. longispina of the Lunz Untersee. Initially, due to meager environmental conditions the animals remain smallheaded; however, in the hothouse they soon developed tall-headed modifications, which would in turn assume the original form upon replacement in the old environment. After two years and 40 generations, the animals produced tall-headed young even when transferred to low temperatures with poor food during egg development. The tall head-height of the animals thus raised therefore represents a deviation from the standard reaction norm of this type. However, when the offspring of the modified animals were placed in the reduced environment, they instead showed the normal reaction for the type (small-headed). Nevertheless, a rather troublesome observation was that the modified reaction appeared after a relatively long time, and it took many generations of experiencing the same influence to achieve a sustained alteration of the head-height reaction.

\section{9-1911}

In this period I resumed experiments with the enhanced environment to test again and again whether eventually the second generation after replacement in ancestral conditions could show that same reaction modification. At this point I have nearly doubled the count of years and generations, without fulfilling my expectations. Regularly the animals resumed the normal head-height again after replacement in the ancestral assimilation conditions. The 
experiments will continue so long as the lines remain simple to propagate parthenogenetically. However, I have rather little hope that a further two or four years will yield a real step forward. At this time, contrary to my earlier optimism, I must question whether a true transmutation (which is to say a modification of the reaction norm) can be effected for this character through cumulative environmental influence.

\section{PREINDUCTION OF HELM HEIGHT THROUGH ENVIRONMENTAL INFLUENCE WITHIN A GENERATION.}

In 1909 I began similar experiments with the tall-helmed Hyalodaphnia cucullata. This type shows a wide range of head-heights, as shown by the pictured plus- and minus-variants (Fig. 1-2). The differences are visible both for the pictured adult animals (Fig. 1) and also in newborn young, independent of external and internal influences (this is methodologically very important).

I was rather surprised to confirm that for this type, which is generally much more sensitively than D. longispina, exposure to an extreme environment for only a single generation effected a modification in not only the next generation (born in the environment), but also the next generation after that. Of course, the parent of this further generation must be withdrawn from the stimulating environment before egg development occurs. The dramatic reduction of assimilation (hunger and cold) proved efficacious in achieving this effect. From the eggs of these animals born "in minimum" come forth young that are and remain small-headed even when raised in the best environmental conditions.

One can also achieve this reduction of assimilation by removing an antenna from the animal. Such animals cannot swim freely, but nevertheless survive while the antenna regenerates slowly, ceaselessly describing circles on

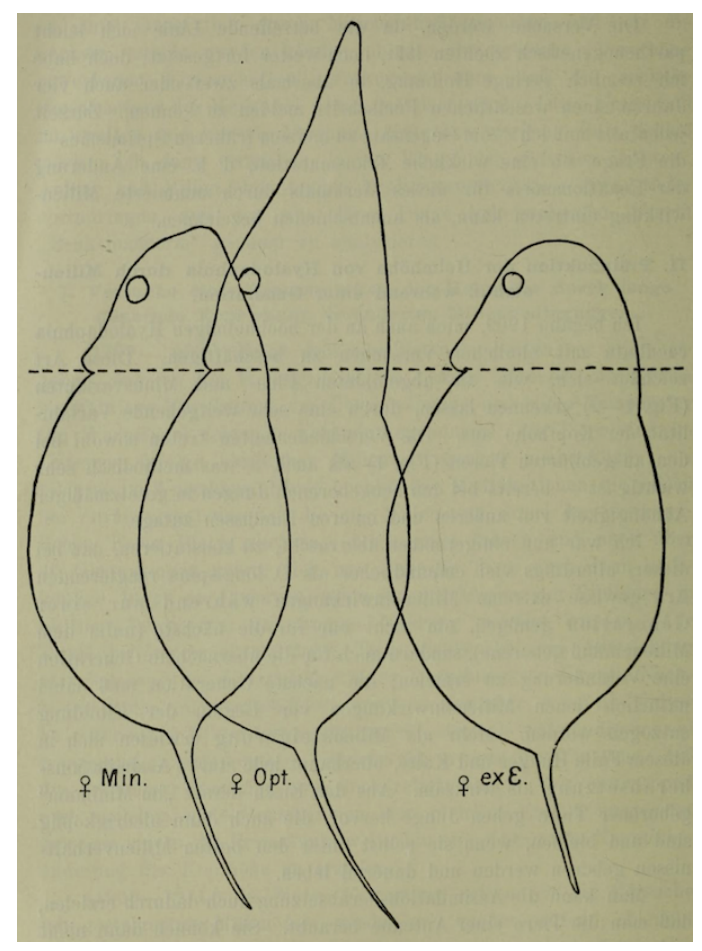

Fig. 1. Limits of helm-height inside a pure line of Hyalodaphnia from Frederiksborg, representing averages (eggbearing 우). At left, two 우 of a middle generation for minimal and optimal culture conditions ("Min.", "Opt.", respectively). At right, one of of the first generation after ephippium under optimal conditions. Scale enlarged 90X. The base of the head is defined by the line connecting the lower edge of the rostrum and the point of contact of the second and third antenna muscles. 
the bottom of the culture vessel 4

Their young are consistently small and have short heads corresponding to the reduced assimilation of the mother's body. Their heads remain thus, even if they are brought up in normal culture conditions after birth. They grow there to fully normal body size and egg production and show no deviation from the average other than in head form.

However, the offspring of these animalsborn in normal culture-clearly show the aftereffects of the meager assimilation under which their mother developed. They are not so small as their parent was at her birth, but nonetheless much smaller than normal for their degree of assimilation.

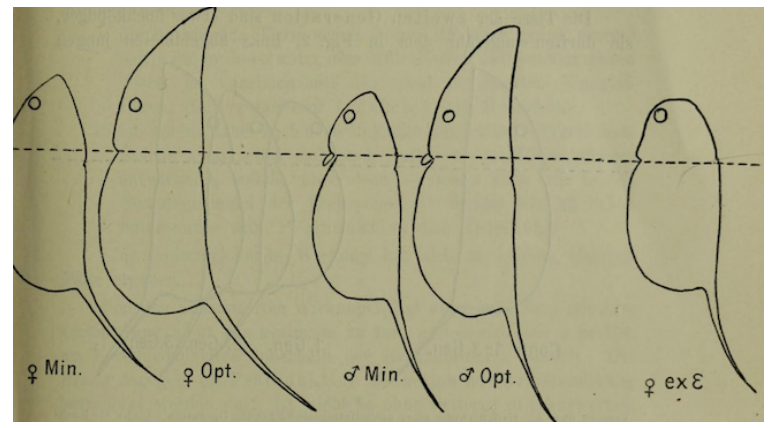

Fig. 2. Limits of helm-height inside the same lines of Hyalodaphnia as in Fig. 1, representing averages (newborn 우 and $\left.\sigma^{x} \sigma^{x}\right)$. For each, one $q$ and $\sigma^{x}$ born under minimal culture conditions ( $10^{\circ} \mathrm{C}$ and scarce food, "Min.") is represented, and also one $\sigma^{\circ}$ and one $\bigcirc$ under optimal conditions $\left(22^{\circ} \mathrm{C}\right.$, rich food, "Opt."). At right, one + represents the average animal born from dauer eggs ("ex $\left.\epsilon^{\prime \prime}\right)$. Scale enlarged 90X. The base of the head is defined by the line connecting the lower edge of the rostrum and the point of contact of the second and third antenna muscles.

In Fig. 3 I represent the mothers in question. The left $q$ represents the similarly tall-headed 우 of the control culture, born to a mother born in an optimum environment (" $y$ ", $22^{\circ}$ and rich Chlorella food), whose offspring are reared in similar conditions.

\footnotetext{
${ }^{4}$ MOP note: I will refrain at this point from making any comments about the existential state of graduate students.
}

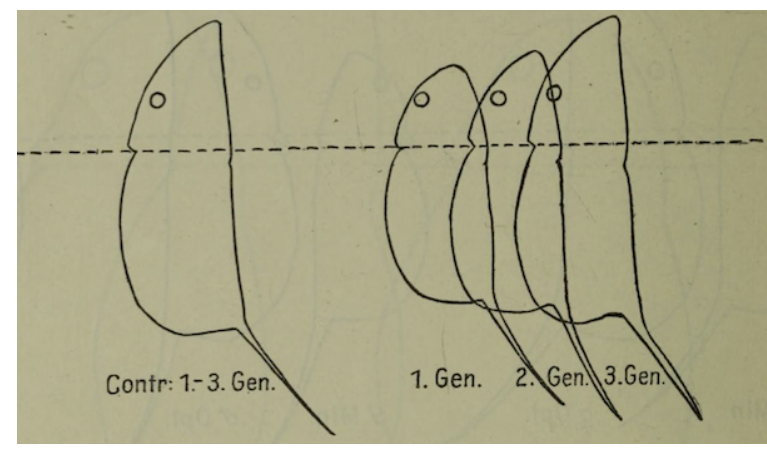

Fig. 3. Experiment upon the effects of an assimilationreducing operation. At left, the offspring of an unoperated control female (all generations alike, "Contr."). At right the first, second, and third generations after the operation. Represented are the average animals of the first clutch of each generation (newborn 우).

Right or left antennae were amputated from some siblings of this $q$, and from one of the $q \circ$ thus manipulated (" $3 y^{\prime \prime)}$ I obtained not only daughters and grandchildren, but also greatgrandchildren (in this experiment sooner or later $o^{7} o^{7}$ and dauer eggs also arise). These three female generations were very instructive. One average female from each generation is shown at right in Fig. 3.

The first generation (which developed in the brood chamber of the damaged o) consists of greatly reduced animals, rather smaller and shorter even than those raised in a minimal external environment (without manipulation).

The animals of the second generation were somewhat more tall-headed, and may be approximately likened to the young "minimum-o" pictured at left in Fig. 2, even though it was born under rich normal conditions of assimilation.

However, the third generation appeared in all respects to be fully normal.

Repeating the operation many times on successive generations did not yield any further result. The influence of assimilation disturbance never reaches further than the grandchildren of the manipulated of , which is to say the children of the animals born under this disturbance. Even in later broods of this second generation the effect is less clear; experiments 
are in progress investigating this.

I obtained very similar results with the progeny of ㅇ afflicted by parasites (Microsporidians), in which the offspring remained free of this assimilation disturbance. This again took the form of an aftereffect up to the grandchild of the affected animal, and no sign whatsoever of an effect on the greatgrandchild (as of yet).

The full results of all these experiments (including the unfortunate and protracted data of the first section) are as follows, including some next steps:

1. I could dramatically alter the specific animals in question through action of the environment (if they are young enough), as well as those animals developing within them under the same conditions, compared to control animals without this influence (induction of helm-height).

2. I could in these cases also alter those generations developing after the cessation of the environmental influence, but whichif only in the gonadal primordia of the embryos-were still subjected to the same influence (preinduction of helm-height).

No operation of the environment reaching further than this is observed in a single case.

The operations thus proven have nothing in the least to do with the modification of types. This is especially true of $1 .$, which is surely an instance of different expressions of the reaction norm. The other question, namely whether preinduction may be taken as the initial step of type modification, is unsettled. But it already appears that this is somewhat questionable. These questions will be discussed more later, in a more favorable case.

\section{AtTempts towards A}

TRANSMUTATION OF SEXUALITY THROUGH LONGSTANDING ACTION OF ALTERED ENVIRONMENTAL CONDITIONS.

Such a case may be found in the sexuality of Daphnia, which is to say the cyclical tendency of their ovaries to produce sometimes parthenogenetic 우 and sometimes $\sigma^{7} \sigma^{7}$ and dauer eggs. This tendency is well-known as an extremely variable character, it is carried out in a somewhat different fashion by each type, and it fluctuates regularly even within individuals of the same pure line. Additionally, like helmheight or other morphological characters, it is dependent on environmental conditions.

I have shown previously (loc. cit.) how sexuality can be treated as a quantitative variable character in some biotypes, although two competing tendencies or genes $5^{5}$ are in play. Both tendencies depend in part on the environment and partially on innate factors. The first of these is the tendency to produce $\sigma^{x} o^{x}$ and dauer eggs, and the second is the tendency to produce pure female-parthenogenetic individuals. Together, these give rise to the regular fluctuations of sexuality in Daphnia. The sexual reaction norm may be represented numerically in the rise and fall of the "bisexual" tendency, expressed in the percentage of egg production overall. (See the curves shown in my earlier work.)

\section{i. 1907-1909:}

Ordinarily, Daphnia cultures become bisexual in normal environments after some number of parthenogenetic generations. However, when I raised cultures of D. pulex, obtusa, or longispina for some time in an optimal environment, I found in some cases that I could hold them longer at low temperatures with poor food before the expected onset of bisexuality appeared. I believed that this must signify a modification of the reaction norm during this

\footnotetext{
${ }^{5}$ MOP note: Woltereck uses the term "Anlagen", which given his earlier statements in the introduction, I will (usually) translate as "genes" hereafter to assist the reader.
} 
months-long residence in optimal culture conditions, because "the later generations thus acclimated lose the ability to react (through sexuality) to minimal food and temperature" (Woltereck 1909).

We maintained these cultures of D. longispina, pulex, obtusa, and cucullata up to the present day, and we continued to analyze their sexuality. These experiments revealed the following.

\section{ii. 1909-1911:}

One portion of these lines (particularly a $D$. pulex from Lake Astroni near Naples) remain exclusively parthenogenetic in their reproduction, and do not deviate from this means of propagation in minimal cultures so long as they can still produce eggs. These animals appear to have become truly asexual (or more precisely unisexual), although I did occasionally observe $\sigma^{\top}$ in the wild Lake Astroni population.

However, I cannot prove that the warmth and rich nutrition during the first year is actually responsible, because at the beginning of the experiment I failed to test the sexuality of these now-asexual lines under different environmental conditions. Thus, it is formally possible that these lines were purely and heritably asexual from the beginning. I have unfortunately been unable to repeat the experiment with newly-collected parental materials, because the Astroni crater has recently been closed off as a royal hunting park. My attempts to obtain permits for collection have been fruitless, presumably because the Daphnia are better employed as game ${ }^{6}$

Daphnia longispina had overall similar behavior; these 3- and 4-year-old cultures show a dramatic diminution of bisexuality. I believed that lasting asexuality was established after the summer of 1910, as none of the three lines gave rise to either $o^{7} \sigma^{x}$ or dauer eggs for 1.5 years. But since that time some $\sigma^{7} \sigma^{\pi}$ were born.

In a related observation, cultures of D. obtusa also returned to bisexuality after they had been fully asexual for approximately 16 months.

\footnotetext{
${ }^{6} \mathrm{MOP}$ note: As far as I can tell up to now, this is Woltereck's only venture into irony.
}

For a full evaluation of the results obtained from both of these types, I would like to defer judgment for the present time; these cultures will be continued.

The behavior of a H. cucullata landrace from the Frederiksborg castle lake proved rather clearer, such that I could thoroughly analyze their sexuality 7 These cultures made me aware of a significant difficulty with my interpretation of my previous results. Namely, we can obtain parthenogenetic offspring from these later, highly bisexual generations that ordinarily terminate the colony's annual cycle. We have known this for some time 8 However, these "yet later" generations do not maintain a highly bisexual tendency, which is what I had assumed. Rather, they return-either gradually or suddenly-to parthenogenetic egg development.

Previously, I was only aware of this behavior in the dicyclic Cladocerans, in which at the end of the first sexual period, surviving ofo (not from dauer eggs, as previously thought by some) renew parthenogenetic egg production. This is likewise due to the inherited reaction norm of sexuality in these animals, with bisexuality increasing step by step from generation to the next, and then through influence of the environment receding once more in monocyclic forms (see the curves given in my earlier arti$\mathrm{cle}{ }^{9}$. Fig. 15).

Once again I would like to emphasize that the animals that ordinarily close out the annual cycle in a sexual state (those of the second cycle under dicycly and respectively those of the single cycle under monocycly) can return once more to the starting conditions of stable, environmentally-insensitive parthenogenesis. This assumes, of course, that the autumnal bisexuality was not of the absolute kind shown by some biotypes. The curves given in Figure 4 attempt to illustrate this phenomenon. These curves represent the rise and fall of bisexual-

\footnotetext{
${ }^{7}$ Über Veränderung der Sexualität bei Daphnien. Experimentelle Studien über Geschlechtsbestimmung. Leipzig 1911. (Internat. Revue d. ges. Hydrobiol. etc.)

${ }^{8}$ MOP note: I added this clarifying sentence.

${ }^{9}$ MOP note: He means the 1909 article introducing the reaction norm.
} 
ity in some pure lines of H. cucullata, which were maintained under middling and (so far as possible) similar culture conditions ("standard cultures"). The bottom curve is most informative (of line $\eta$ ), because it continutes across all four years 1908-1911. The decline of bisexuality after each period of sexuality proceeds even without the action of warmth and rich food. Altogether, the autumnal sexuality declines in those cultures that simultaneously experience the onset of cold and food scarcity in the wild, where these precipitate the end of the colony each year ${ }^{10}$ The standard cultures represent on average a superior grade of environment to that experienced by these animals in the wild.

Notably, there is not a total cease of bisexuality (as I previously reported), but rather-at least in some cases-an unmistakable progressive diminution. This diminution takes the form of an increasingly long interval between one sexual period and the next (apart from some intermediate starts towards bisexuality). At least the last period (at the end of 1910) appears much less prominent than, for example, the period at the end of 1908. I await further data regarding culture $\eta$ with some anticipation.

In the meantime, it is difficult to see any relationship between transmutation and the alterations experienced by sexuality over the course of a normal (annual) cycle. (The generations that we deal with in cultures could be seen as "meta-cyclical" relative to the normal cycles.) If we must postulate a "transmutation" of the reaction norm for observations in culture, then this raises the question of whether the attenuation of metacyclical periodicity (as seen in $1909,1910,1911)$ truly represents a modification of the normal cycle (1908). This question cannot be decided at the present time. And so we must reserve judgment and continue the experiment for this chapter, as for the first.

\footnotetext{
${ }^{10}$ Very occasionally, starving 우 can be found in winter under the ice.
}

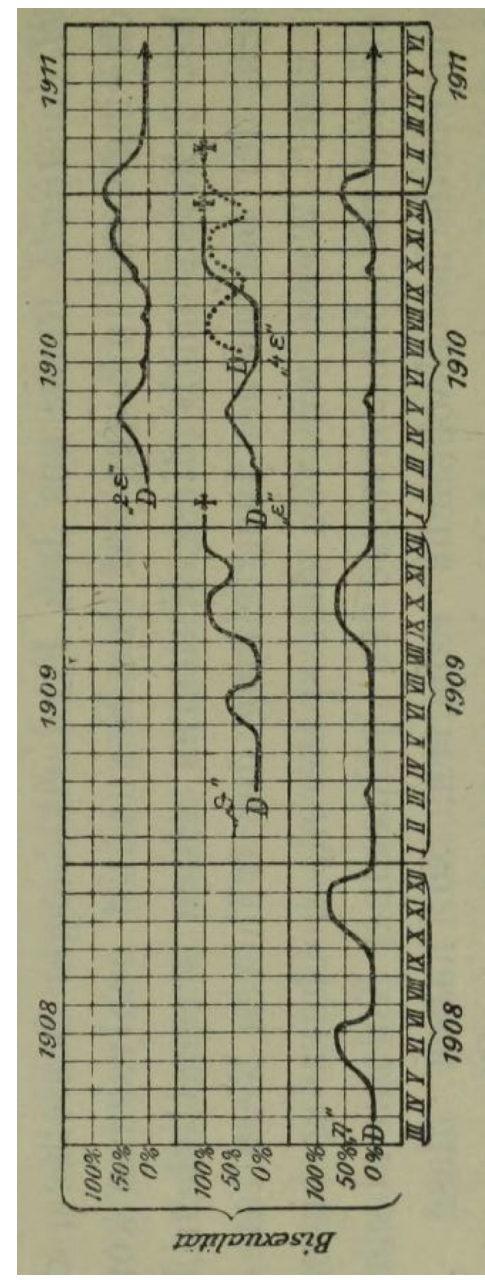

Fig. 4. Sexuality curves of five "normal cultures" of Hyalodaphnia. The cultures arise from dauer eggs (" $D$ ") and end either in absolute bisexuality with the death of the last sexual animal $(y)$ or are ongoing $(\rightarrow)$.

\section{PREINdUCTION OF SEX IN} HYALODAPHNIA IN ONE GENERATION,
THROUGH ENVIRONMENTAL ACTION
DURING THE SENSIBLE PERIOD.

The "clear behaviors" of H. cucullata that I describe above were the fruit of my initial analytical treatment of the inheritance of acquired characters. Specifically, they allow us to dissect this problem more finely than was previously possible, and specifically the phenomenon denoted as preinduction.

Regarding what I have already shown for 
morphological characters such as head-height, we may see the following as probable. The preinduction of a character is not necessarily a change of the reaction norm, and therefore not necessarily a transmutation. This also proved to be the case for the determination of bisexuality. The influence of sex on the next generation is surely the inheritance of an "acquired" = "compelled" = induced character by the meaning of many authors. Nonetheless, we name this mechanism a preinduction, because the induction of a generation earlier determines the character on display, and therefore the induced condition must be at least slightly heritable.

However it is equally certain that this inheritance of acquired characters has not the least to do with modification of the type, because these same sexual traits are hallmarks of the type. (Only the diminution or loss of the male sex is in question as a possible transmutation, but that does not concern whether we can influence the sex from one generation to the next.)

The time-point of influence may be found in a transition of the eggs to a new state, which occurs in the brood chamber of the ovary. At this point the sex of the eggs will be determined, just as the color of a butterfly wing is determined when the pupal wing primordium has progressed far enough for pigment development to begin. In both cases, the influence of external factors (such as generation or clutch number in Daphnia) becomes effective at this time. Apart from this, the time of internal character determination is also a period of sensibility to external influences. In other words, the activation of genes ${ }^{11}$ and the sensibility towards induction coincide in time.

Preinduction. Preinduction also demonstrates a defined sensible period. As is well known, the germ cells of Leptinotarsa (Tower) are only sensible for a short time to influence on the coloring of later generations, specifically during the creation of a new batch of germ cells in the beetles. Only during this sensible stage may preinduction act on the germ cells.

\footnotetext{
${ }^{11}$ MOP note: he again uses the term Anlage here where I render "gene".
}

Preinduction by temperature in the germ cells of some Arctia pupae (Fischer) is similar. In this particular case the sensible period coincides with the time of (normal) induction. In contrast, in the Colorado beetle the time of preinduction sensibility is separated from the time of induction sensibility. Some have spoken of "parallel induction" in butterflies. Likewise, if the sex of the eggs is female, but their descendants are already determined to be male ("male-producers" of rotifers and Daphnia), one can name this "simultaneous induction". Finally, in Leptinotarsa (and again with Daphnia), we observe that "separate induction" also occurs. This last example betrays the fundamental point: induction and preinduction are two independent reactions. Regardless of whether they occur at the same time and have the same outcome, or at the same time with an opposite outcome, or if each runs its own unique course (as for Daphnia).

The facts are rather more complicated in Daphnia than in the previously known cases of preinduction. According to our best current knowledge, there are three different periods or stages of preinductive sensibility. Two of these periods coincide temporally with induction sensibility (though not always in outcome). I have only investigated the preinduction of feminine germ cells; these are related to the sex of the expected next generation in the following fashion:

1. The final ovarian eggs can be preinduced at the same time that their own sex will be decided ("determined") and eventually influenced by external sources ("induced"). We demonstrate that influences restricted to this time can still express themselves in the sex of the following generation. For this expression, it is not necessary that such influences also induce the sex of the egg exposed in the experiment. For example, it is a common case that we cannot deter (through hunger and cold) a batch of eggs from becoming totally or principally female ${ }^{12}$, but these +9 themselves produce

\footnotetext{
${ }^{12}$ In sex determination, the external factors interfere in
} 
$0^{7} \sigma^{7}$ and dauer eggs when we raise them under optimal conditions.

2. At approximately the same time, a second preinduction process may also occur. The undifferentiated cells of the germ epithelium are themselves ofo, but if at the appointed time induction and preinduction occur in the mature egg, they will later develop in part into nurse cells and in part into egg cells to build the next batch of eggs. It is only then that the influence of hunger and cold experienced by the ofo in question show themselves. These later operations will coalesce into the development of $\sigma^{x} o^{x}$ and dauer eggs, even if the ofo in between is brought into conditions of rich assimilation. A precondition for this result is that preinduction meets the right epithelial conditions (in other words, that the germ stock is just preparing to create a new batch of eggs, especially because this is often not the case in starved cultures). Further, it is necessary that the animals not be in a state of "absolute parthenogenesis" or "absolute bisexuality", because they cannot be influenced while in these absolute states.

3. In turn, there is a third independent stage sensible to preinduction: when the gonadal primordium in the embryo is in the quiescent dauer state (in the ephippium). Here induction is not possible, because dauer eggs are well-known to give rise to females exclusively. However, in a Hyalodaphnia from Frederiksborg, the

the internal determination process if the competing Anlagesubstances (genes) of $q$ and $\sigma^{7}$ sex are found a priori in equal measures in a given circumstance. Sexuality varies periodically in Daphnia with the prevalence of masculine or feminine autogenous determinants. Expressed differently, sexuality shows alternating recessivity $[M O P$ note: it goes without saying that Woltereck is not using a modern definition of "recessive"; it appears that he is speaking of what he calls "dominance shifts" elsewhere in this text]. In the transition from one state to another, cases occur where preinduction is possible but not induction, and the converse as well. If we could not thus separate preinduction and induction experimentally, the analyses regarding preinductive "sex determination" of different types sketched out here would hardly have been possible. gonadal primordium (composed here of only a few cells; Häcker's kernel group ${ }^{13}$ proved sensible to a preinductive temperature treatment. From the germ stock of the ex ephippio female, purely parthenogenetic of appeared, which from that point also produced $q \circ$ themselves. Through a warm treatment, such females gave rise to $0^{7} o^{7}$, and further-contrary to all reason-these same females proceeded to dauer egg production 14

That these three quite distinct cases concern similar processes in the plasma is suggestive. It appears to me difficult to deny the importance of these processes, because the internal periodic modifications of the sexual tendency likewise appear to proceed in these three grades. I gave the necessary notice that sexuality modifications in dauer can proceed autonomously through preinduction (by temperature). For the other two cases, it suffices to recall that autonomous sexuality modifications are evident from generation to generation or brood to brood. For more information I refer to my most recent work.

I wrote above that sensibility towards induction coincides with the activation of the genes ${ }^{15}$ (for sex, for wing markings, etc.). In my recent work on sexuality (loc. cit. 1911) I expressed the opinion that the sensibility towards preinduction must coincide with some "maturation" process of the genes. This leads to some final outcome resulting from which genes 16 attain the "active" state and which remain latent. For the experimental study of such processes, Daphnia appears to be betterappointed than other organisms, because they

\footnotetext{
${ }^{13}$ MOP note: literally Binnenkerngruppe; I could not find a previous translation of this term.

${ }^{14}$ I reached the same result for this landrace by treating the ephippia with cold and dry conditions one month before the normal time (in July). In these last cases inner (autonomous) processes tied to a certain course of time appear to be responsible for the alteration of sexuality. It is not possible to merely speak here of "over-maturation". In each case however the comparison with R. Hertwig's masculine induction of over-mature frog eggs comes to mind.

${ }^{15} \mathrm{MOP}$ note: Anlagen.

${ }^{16} \mathrm{MOP}$ note: Anlagen.
} 
alternate between different states (labile, nonlabile but with equal valence, or pre-valent and recessive ${ }^{17}$ with very distinctive periodicity. Perhaps through the testing of these phenomena we will achieve a deeper understanding of the processes underlying induction and preinduction. For the central question concerning us here, regarding the behavior of preinduction and transmutation, understanding of these processes is essential.

\section{HOW DO PREINDUCTION AND INDUCTION PROCEED?}

In answering this question, we must be particularly careful in examining the experimentally determined facts. Moreover, we must in particular scrutinize the conclusions or opinions that may be arrived at in interpreting them. Such working hypotheses are of course indispensable, if we desire to come closer to understanding the answers to our questions in such an intricate domain.

First, it is true that induction and preinduction appear in numerous instances, with quite satisfactory positive findings in investigations using environmental perturbation.

Further, it is true that these two phenomena concern an intervention of external factors in those reaction processes that affect the development of visible qualities. We observed at the beginning of our studies that essentially all (variable) characters can be controlled through these reactions.

And indeed, the visibility of a character depends more or less on the reaction between its plasmic substrate and its heritable representation and determinants (which we will refer to as "genes", as the briefest and most general

\footnotetext{
${ }^{17}$ MOP note: this is a bit confusing, as Woltereck appears to be using the cognate words for "equivalent" and "prevalent" to refer to the "valence" (activity level) of genes in an analogy to chemistry, and is also using "recessive" in an unfamiliar sense. The three states listed appear to correspond to the three enumerated above.
}

of the available expressions). In this reaction process the environment acts in an "inducing" fashion.

In which reaction preinduction intervenes is not easy to say. It is presumably in a much earlier process, in which these genes are likewise concerned. Previously (loc. cit., 1911), in trying to explain my experimental results, I hypothesized a process by which genes shed their latent (constrained) state, in which they must first pass through a not-yet-activated state before making their effects known (e.g. expressing phenotypic characters). I named this intermediate state "activation competence".

The stage of induction sensibility coincides with the process of gene-activation, while I opined that preinduction sensibility is confined in time to a period merely preparing for activation competence.

A further question is then how the factors of the milieu intervene in these processes. We will now attempt to answer this question for the alternative characters.

\section{i. Induction and preinduction of alter- native characters.}

We now deal with-using as an example the scale coloration of Vanessa levana-at least two visible qualities, which are thus heritable as alternative characters, and therefore also with at least two genes ${ }^{18}$, and finally with at least two reactions.

Then the visible result of the one reaction (Gene A) is Color A, and that of the other reaction (with Gene B) Color B. Upon activation, these reactions occur in the wing substrate, whereas upon the appearance of activation competence they instead occur in the germ cells.

The fact that only one of the two inherited

\footnotetext{
${ }^{18}$ MOP note: In this we can see a confusion of Mendelism. We might today understand such a simple Mendelian ("alternative" in that it is qualitative and not quantitative) character to be defined by two alleles of a single gene (this terminology was introduced by Bateson and Saunders by 1902 and is used in Johannsen's book, with which Woltereck showed some familiarity). It is sometimes accepted to refer to the two alleles as independent genes, but Woltereck does not seem to mean it in this way.
} 
characters becomes visible seems to show that only one of the two reactions will complete, in general. The other reaction will then be incomplete or remain unexecuted. If we simply say that this reaction is inhibited, then we probably state the actual case in the most hypothesisagnostic sense.

The further conclusion, that this reaction inhibition is caused by environmental action, now lies very near. We want to tentatively approach this conclusion, to more deeply understand the issue at hand.

Therefore: action of the environment brings about the inhibition of one of the two alternative reaction courses. This has the consequence that only the other reaction is brought to its conclusion, and therefore the other quality is actually realized.

Altogether, we must distinguish between two actions: induction means inhibition of activation, and preinduction means inhibition of the transition to activation competence. We can name these processes "activation inhibition" and "maturation inhibition".

If we ask further where these inhibition processes can subsist, we come to truly hypothetical ground. Nonetheless, it is surely clarifying to cover such ground in answering our questions.

Towards this end, we desperately need a closer understanding of the nature of the oftmentioned genes, of which we at present unfortunately only know-and that only since the rediscovery of Mendel's work-that they behave as indivisible heritable units determining certain characters ${ }^{19}$ with greater or lesser effect.

\section{9}

They do not consistently determine singular organs or cell territories, as some assumed earlier. Rather, the Mendelian units (even those known to the man himself) seem to affect the body at large. Of course, for my understanding this is no hindrance. If a gene of the germ cells is later active in many body regions, then a reaction responsible for the determination of each cell occurs as an interaction between the plasmic substrate and the active portion of the gene substance. The understanding of the animal body may (at least in some cases) not be advanced through this means of investigation, as we observe from Johannsen and the strict Mendelians [MOP note: what we now call the Mendelian-Mutationists, including also De Vries
First, this understanding must accommodate the few rules that we know about the physiological chemistry of the substances in question. Second, it must agree with our experimental knowledge of the organisms.

Bredig wrote in his work about "Inorganic Catalysts" that inorganic catalysis and its operation could be taken as a model for understanding organic enzymes (whose synthesis remains unknown to us) and their action. Of course, the differences of "organizational complexity" (expressed biologically) of the bodies under comparison are immense. Nonetheless this path of comparison has been very fruitful for the study of reaction catalysis. Our knowledge of the conditions and scopes of catalytic activity are astonishingly expanded since Berzelius came to understand catalysts.

At this point, I must re-emphasize the similarity (in behavior and mode of operation) between the organic catalysts (enzymes) and those character-determining substances of a cell or a developing organ (genes). In such cases (for example, the primordia of pigment development for some insects) these relationships are quite close. However, the general circumstance of inherited genes that effectuate a broadly chemical and formative alteration from generation to generation at some minimal level without themselves becoming altered or depleted might suggest a similarity with catalysts. The "organizational complexity" has already climbed very far from its origin, as it now concerns not only the cleavage or synthesis of chemical bonds, but also the activation of specific processes in the development of organismal structure 20 Similarly it will be ap-

and Bateson among others.] when they completely abstract morphological characters and units (organs, cells) and only consider genetic units. The morphological units must maintain their meaning, as they provide the genes their potency and also their substrate. We know the genes only through the visible results of the reactions that represent them. It is certainly understandable, even if regrettable, that the newest and most successful direction for biology, resting upon this analysis of heritability, jettisons the analysis of bodies as obsolete. One should not let this serve as discouragement.

${ }^{20}$ MOP note: Garrod's (and then Bateson's) influential work on inherited errors of metabolism was published in 1902, so 
propriate and useful in this context to repeat the helpfulness of having a simple system as a preliminary model for our more complicated one.

Yet another set of phenomena and conceptions from physiological chemistry may now be repeated (most emphatically those of von Rhumbler ${ }^{21}$ for consultation towards explaining the facts of heredity. In connection with Ehrlich's famous side-chain theory, the theory of antigens and further work in immunology may illuminate many things.

$I^{22}$ would be good for our endeavor to find a viable chemical model of the gene etc., as enzymology and immunology have found in each other, as for example Oppenheimer ${ }^{23}$ has thus described catalysts as a specific type of antigen. We can therefore take, for the conception of our model, a physiological concept from catalysts and a structural picture from the antigens.

Without fully formulating such a conceptual model (this would be to go too far very prematurely), I want only to remind us of certain ideas in the studies of catalysis and antigens that may be helpful. For some such ideasrather freely adapted and simplified-this borrowing of ideas leads to the following conception of our present data:

1. The gene consists of a "framing nucleus" ("productive nucleus", "scaffolding nucleus' ${ }^{24}$, which at precise times will produce "ergophores" (specifically, when it reaches the "enzyme stage"). Together, these represent the chemical and formative activities of the gene. Next, out of the scaffolding nucleus the "haptophores" are created. These are responsible for conjugation with other substances, and can anchor

there is plenty of opportunity for the development of such ideas. However, the combination of enzymatic action with the idea of gene regulation appears to be quite novel here, though apparently unappreciated at the time.

${ }^{21}$ Intern. Zool. Congr. Boston 1907.

${ }^{22}$ MOP note: these following five paragraphs are printed in smaller type in the original.

${ }^{23}$ Die Fermente und ihre Wirkungen, Leipzig 1910.

${ }^{24} \mathrm{MOP}$ note: Stammgerïst in the original. the scaffolding nucleus to "inhibiting" substances that the gene cannot influence (this puts the gene into a latent state). These haptophores can also anchor the scaffolding nucleus to those substances that are required for its activity.

2. If the gene is anchored in such a way that it is inactive (latent), it resembles a "zymogen" (proenzyme) or a "zymoid" ("enzymoid"). In catalysis research, the first term indicates latent but activatable catalystlike substances, the second similarly indicates catalyst-like substances that have however become latent (following some period of activity), but are no longer activatable $e^{25}$. We require the further assumption that inactive zymoids can become active zymogens.

3. Gene activation occurs as follows. Specific materials that may be described as "activators" bind proenzymes by their open haptophores. This can be done by either chemically simple substances (e.g. acids or metal salts) or perhaps by other albumen bodies ("kinases"). The binding or inhibition of the genes through so-called "plugging" of their haptophores occurs under the influence of specific substances that are called "paralyzers" in the catalysis literature, most importantly the so-called natural "antibodies" (detached haptophores). A further extension of our theory along these lines must be ceased for reasons of economy.

For this "enzyme theory of inheritance" sketched out here, we therefore confine ourselves to the following statements:

- The gene-molecule is composed of the scaffolding nucleus, haptophores and ergophores.

\footnotetext{
${ }^{25}$ For simplicity, we shall ignore for now a conspicuous character of known zymoids, that they can be inhibited by antibodies from carrying out their catalysis. It is nonetheless possible that genes mutually influence each other through the creation of antibodies (paralyzers). This potential behavior may play a role in this valence relationship.
} 
- The three linked states of zymoid, zymogen, and enzyme are possible states for each gene-molecule.

- Genes are activated through binding of their open haptophores with an activator and the consequent production of ergophores.

- Genes are inhibited through blockage of their haptophores by some paralyzer ${ }^{26}$

If we turn once more to our experimental results, we next must confirm that each Anlage (gene) does not proceed in two states (latent and activated) (as is commonly assumed ${ }^{27}$, but rather in three clearly distinguishable stages:

- First stage: The gene for a bodily character is latent and not activatable (the common state in the germ cells, during fission etc.).

- Second stage: The gene is latent but now activatable (shortly before determination of the gene substrate).

- Third stage: The gene is activated (the character is visible).

These states are connected by two transition periods, which have been shown to be temporal in character in both Leptinotarsa and Daphnia (see page 9). These critical or influenceable periods are as follows:

- Beginning of the transition from first to second stage above ("maturation"): the period of preinduction sensibility. A preinductive "maturation inhibition" is possible.

- Transition from second to third stage above ("maturation"): the period of induction sensibility. An inductive "activation inhibition" is possible.

$\mathrm{I}^{28}$ we apply the conceptual model given above to these findings, we can say something like the following:

\footnotetext{
${ }^{26} \mathrm{MOP}$ note: After this point the original text returns to the normal, larger print size.

${ }^{27}$ MOP note: it is not clear (to me) who then assumed this.

${ }^{28} \mathrm{MOP}$ note: the following 10 paragraphs are again in smaller print.
}

- First stage ("zymoid"): the gene is a scaffold that lacks ergophores, and is bound by its haptophores to some other material that inhibits activation. The genes exist thus in the germ cells, etc.

- Second stage ("zymogen" or "proenzyme"): the gene is a scaffold that lacks ergophores, and whose haptophores are not bound ("blocked"). These unbound haptophores stand open to the influence of appropriate activators. This is the state of the genes at the time of "gene activation".

- Third stage ("enzyme"): the zymogen (or proenzyme) is made functional through binding to the activator, leading to creation of the effectual ergophores. The enzymes or catalysts thus generated are not alterable and participate in the reaction mechanisms of developing cells and organs, which they serve in determining.

Using this conceptual model, we can answer our question regarding how inhibition acts in preinduction and induction.

Preinduction of an alternative character: One or more genes are inhibited through influence of the environment, such that they transition from their normal zymogen state capable of activation into a zymoid state. This transition remodels an integral element of ordinary germ cell maturation. From the mere fact of preinduction we must conclude that for each of the present genes specific conditions must be fulfilled to reach the second state. If these "maturation" conditions (for example temperature) are not fulfilled, then only the other genes are "open". Thus it is decided from that point that later, only those other more "mature" genes can be activated.

If on the other hand these conditions are satisfied for two or more genes, we will have to reckon with more active genes, among which some final decision must be reached.

This decision can be reached if one gene among the others triumphs through its greater "valence". We could compare this valence to a 
greater affinity for the activator (or a greater number of open haptophores?), if we can imagine a common activator (acids, kinases?) ${ }^{29}$ for these similar genes. Then the activators are concerned with the prevalent (dominant or epistatic) genes, and the recessive or hypostatic genes remain empty. These empty genes remain latent, and through other sorts of binding of their haptophores they once more take the zymoid state, or they decay.

Alternatively, the competing open genes are equivalent, or rather they have similar affinity for the activator. In this case, the induction provides the decision: from the competing proenzymes, one will be activated whose affinity for the activator is not inhibited by the environmental treatment (temperature etc.).

Thus, differences of valence among genes may have quite different causes. For recessivity (for example) it may be either induced depletion of activation (affinity) energies that may have been originally equivalent to others, or from preexisting inferiority of affinity energy the gene may similarly completely fail to activate as a consequence of preinduction 30

I hope it is sufficiently clear that these reflections on the mechanisms of the two inhibition processes are purely hypothetical, whereas the existence of the two inhibitions is experimentally demonstrated.

The quantitative (rather than alternative) differences that we observe are similar to differences that can be effected via preinduction and induction, as seen in Section II. These differences exist between individuals of a biotype as well as between different biotypes. It is however not yet known whether these differences arise in nature through similar inhibition processes.

\footnotetext{
${ }^{29}$ One can also make a case for other causes of valence changes, as I have (loc cit. 1911, S. 110). If one assumes that each individual gene has a single activator or if-perhaps closer to the original teachings of catalysis and antigensspecific paralyzers (antibodies) are principally responsible. The creation or non-creation of these inhibiting or activating species, or their different quantities, would then decide prevailing valences of the genes.

${ }^{30} \mathrm{MOP}$ note: after this paragraph normal text size resumes in the original.
}

\section{ii. Induction and preinduction of non- alternative (quantitative) characters.}

Our observations of induction and preinduction, and also our model scheme of the nature of genes, can be taken as a reasonable basis for the behavior of alternative characters (sex, wing pigmentation), because these reaction processes feature rather clear eitheror behaviors in their results. Additionally, the present theoretical ideas regarding the causes of trait determination are predominantly based upon examples of alternatively-heritable traits.

These ideas have found their most incisive expression in Bateson's famous "Presence and Absence" theory ${ }^{31}$, regarding whose great significance and fruitfulness there can be no doubt. Nevertheless, the daily occurrence of findings of "dominance shifts" and related phenomena (Häcker, Plate, Lang and others) make it ever-less-likely that recessive characters signify a lack of genes, or at least that this cannot be the final word. Surely, it cannot be true in the cases where an alteration of gene-valence is evident. We know of such cases where the alteration is autonomous, through preinduction, through induction, and lastly developmentally delayed in the final organism ${ }^{32}$

Particularly for the sex genes, the presenceabsence hypotheses are easy to follow ad absurdum. At least, to sustain Bateson's theories in such cases, any attempt at a hypothesis would be impractically complicated.

Therefore, in both my past and current work I have taken the position that each heritable

\footnotetext{
${ }^{31}$ MOP note: See Swinburne (1962), Annals of Science, for more information on this swiftly-discredited theory. In brief, Bateson supposed that Mendelian characters were defined not by the present notion of "alleles" of the same gene, but by variation in the presence of genes; briefly, where a gene is present, a phenotype is observed, whereas in the absence of the gene no phenotype is observed.

${ }^{32}$ It is not clear whether this last case can be understood as singular, recalling as a familiar example the delayed pigmentation of the recessive-white axolotl (Häcker). These cases may concern autonomous alterations of valence, or a type of "postinduction". Further, it could be that the already-determined cells belatedly become pigmented, or they can be replaced or displaced by other pigmented cells. One could wish for a more stringent analysis in the applicable cases.
} 
gene is present, whether dominant or recessive, and from this assumption my views regarding induction and preinduction are ultimately derived.

It remains to be seen how these views agree with the behavior of the various quantitative or continuous traits that differ across individuals (and races), which do not show discrete trait genes or trait levels.

Such traits include, for example, the ear length of different rabbit races (Castle) or the helm-height of Daphnia biotypes (Woltereck). The first is rigorously determined through a great number of experiments. The second is known through a few experimental results for which it may at least be said that no segregation through crossing appears; when intermediate forms appear, they are certainly inherited.

To salvage the assumption of "stable genes" for rabbit ear length, Lang ${ }^{33}$ presented an analysis postulating an assortment of three genes with cumulative effects, in which each gene increased ear length by a defined amount (40 $\mathrm{mm})$. He supports this with an experimental finding of Nilsson-Ehle demonstrating various numbers of Mendelian genes for black grain color in oats (two genes) and red color of wheat grains (three independent genes).

An application of these ideas to the helmheight of Daphnia is naturally possible. One must then assume that there are maybe $9 \mathrm{cu}-$ mulative genes acting, in the activation of the large helm (Fig. 5b) to a mean value of $90 \%$ (relative to mantle length). For the development of the $50 \%$ helm (Fig. 5c), only 5 genes must be activated.

Let us say that we observe both of these helm forms in a pure line under similar environmental conditions. Specifically, we observe the large helm in the sixth generation after ephippium, while the small helm is observed in the second generation after ephippium; we must therefore assume that in the last case four genes lie latent that can be activated by the

\footnotetext{
${ }^{33}$ Zeitschrift für induktive Abstammungslehre. Bd. IV. 1911. There the further literature, notably the new textbooks of Häcker, Goldschmidt, or Baur, is available in abstracted form, so I will not discuss it here.
}

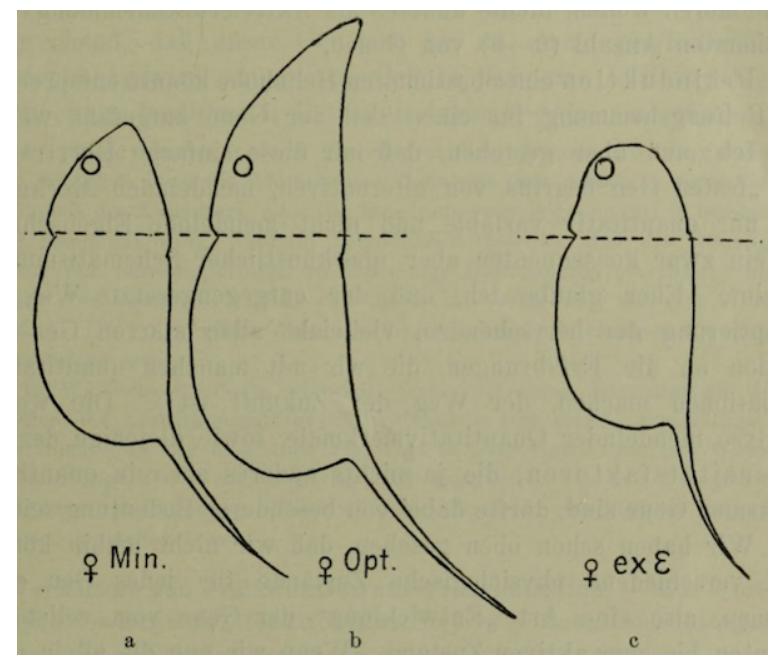

Fig. 5. Helm-height variation in a pure line of Hyalodaphnia (newborn + +⿱). $a$ and $b$ are animals made different by induction in the sixth generation; their helmheight amounts to $52 \%$ of mantle length under minimal culture conditions (a), or $90 \%$ under optimal conditions (b). c: small-headed of of the first generation $(50 \%)$, which remains small in optimal culture and produces small-helmed young.

sixth generation. If in later generations there is eventually a reduction of helm height in the same pure lines, there would then be a deactivation of some such genes. The induction of a certain helm height would (if we choose to combine this fiction tentatively with our ideas from above) be nothing other than the activation inhibition of a certain number (0-8) of genes.

Correspondingly, preinduction of a certain helm height could be seen as a maturation inhibition of some set of genes.

However, I must admit that this simple application of the "stable gene", while consistent, seems to me a rather over-artificial schematism. It applies the behavior of alternative Mendelian traits to variable quantitative nonMendelian characters. I believe that the opposite path is the way of the future, by which I mean adapting the prevailing (altogether too rigid) gene conception to experimental experience, while allowing for some quantitative 
variation 34 The further analysis of Mendelian quantitative traits will be especially significant here, as well as the study of so-called intensity factors, which are nothing different from genes with purely quantitative activity.

We see above that we cannot assume three different physiological states for each gene, or its consequent "development" of the gene from a completely latent to a completely active state. If we make the more attainable assumption that a gene or gene-complex will be inherited for a unitary trait like the ear length of a buck rabbit or the helm-height of a Daphnia race, then we can still arrive at a very simple conception of induction and preinduction.

Induction of a particular helm-height then signifies an inhibition of gene activation to some specific amount. Or rather, if we see a full helm height (for some race and generation), that signifies full activation of the gene in question.

Correspondingly, preinduction of a particular helm-height signifies, that our gene will be only activatable to some certain grade, that its activation competence is designated through inhibition.

In 35 the sense of our conceptual model given above, induction will affect the affinity of the zymogen to the activator (or the nature of the activator, or the generation of ergophores). On the other hand, preinduction operates through a piecewise opening of the zymoids, such that

\footnotetext{
${ }^{34}$ This entire section is a deliberate "confusion of phenotypes with genotypes" (Johannsen), which is to say an attempt to reunite the various differences that we observe for a variable trait ("genotypic" = autogenous, "phenotype" = ectogenous) with the action of (or collaboration with) the determining substances (genes). The modifying environmental influence could interfere in the reaction between substrate and gene through an alteration of the activation energy of the latter. Such an effort, which will be so aggressively condemned by the prevailing direction of Mendelism, appears to me to be inevitable in my heretical conception of the genes (as substances of constitutionally variable energy), and particularly from the reaction norm concept. Whether it should come closer to that dominant direction in the form sketched out here is a different question. Its basis lies particularly in the findings about inducible shifts of dominance of the sex determination genes.

${ }^{35} \mathrm{MOP}$ note: Text again returns to small print for the space of two paragraphs in the original.
}

some haptophores remain "blocked" and only a certain number of them are free to bind the activator. The consequences would thus be reduced activation and reduced enzyme synthesis.

In reality, in addition to all this, the mode of action of the environmental factors may be manifold. It may be that actual catalysts have narrow but diverse dependencies on temperature and other conditions for their appearance and action. Here many interesting parallels may arise ${ }^{36}$

\section{BEHAVIOR OF PREINDUCTION WITH REGARD TO TRANSMUTATION. TOWARDS FURTHER ANALYSIS OF THE "REACTION NORM" CONCEPT37; FOUR REACTION CATEGORIES.}

The exposition in the fifth section may seem to have led us away from the actual subject. Nonetheless, we must attempt to pose some ideas regarding the nature of preinduction if we hope to apply its behavior towards solving the problem of transmutation. This is doubly so for the question of type modification through the action of the environment (the "inheritance of acquired characters").

My ideas about induction and preinductionas inhibition of maturation or activation of

\footnotetext{
${ }^{36}$ MOP note: Text resumes normal typeface.

37 This term of mine from 1909, for the inheritance of variability in a character (see page 148 of the original), was discussed by Johannsen (loc. cit.) and set up as parallel to his famous conception of the heritable "genotype". In 1909 I also wrote "The 'genotype' (the genotypical foundation) of a quantitative trait is its heritable reaction norm" (loc. cit., page 136 in the original), and I considered it important that Johannsen accepted this parallel. By all accounts, Johannsen subsumes the term into something more general, namely as the norm of all reactions (characters) of an organism, while I mean by the term that each single variable trait has its own specific reaction norm. I handle the differences, parallels, and misunderstandings between myself and Johannsen in a different work. [MOP note: I have not located the work he refers to. It does not appear to be the 1911 paper in the Hydrobiologische Referat.]
} 
genes-may be accepted or rejected. They have nothing to do with the independent conclusion that preinduction (a part of the regular inherited reaction norm) and transmutation (alteration of the reaction norm) are quite different processes.

This last thesis appears to me to be established under all circumstances, while my theory of induction and preinduction (and gene structure) are merely tentative attempts to give these physiologically indistinct things that we call "factors" a steadier meaning and basis, by considering them in terms of enzymatic action.

We may at least be certain that preinduction (particularly of sexual traits) can only be a physiological reaction process whose course is ordinarily predestined. This process must be repeated whenever the correct causes meet together at the correct time. Consequently, this process is part of the standard hereditary complement in those Daphnia where it is observed. It has no more to do with the heritable alteration of the biotypes than induction does.

For the preinduction of other traits of the animal body ${ }^{38}$ the situation may be similar, although it cannot be so clearly decided whether preinduction initiates an alteration of the hereditary component (reaction norm) or not. In any case, we will be able to understand preinduction as transmutation (as we might from the many experiments of Standfuss, Fischer, Kämmerer etc.) when the environmentallydependent changes persist for many generations after the environmental stimulus is removed.

This may be the case for Leptinotarsa preinduction; Tower observes a high grade of heritability $(62 \%)$ of the preinduced modification. It is true that the situation is rather complicated.

\footnotetext{
${ }^{38}$ There is no fundamental opposition between the determination of sex and other traits. That will be shown by the crossing experiments of Correns, Castle and others, as well as my investigations on the causes of sex determination in Daphnia. It is shown (loc. cit. 1911) that all the contradictory conjectures regarding sex determination (direct influence of environment, heterochromosomes, etc., nucleoplasmic relationships) can only have a secondary meaning, while the actual causes of sex must be sought in these same "anlage substances" (genes) that we put so much stock in for alternative characters.
}

Leptinotarsa types show some hidden polymorphisms (perhaps like Oenothera after earlier hybridization: ${ }^{39}$. As a consequence, the "mutations" regularly achieved through preinduction were exceptionally difficult to replicate in subsequent reports.

Weaker still are the "heritable" color changes achieved through preinduction in butterflies, amphibians, and reptiles, as well as the extremely interesting "modifications of instinct" in Kammerer's recent publications. If they only last as long as the next generation following removal of the environmental stimulus, we may by no means call them transmutations. Rather, they are only observations of predetermined reactions included in the reaction norm.

The results of my analytical efforts seem to reveal that the concept "inheritance of acquired characters as type-changing factors" must be somewhat narrowed, in that it must be separated decisively from preinduction ${ }^{40}$ The previously formulated idea of the reaction norm will nonetheless help us to understand the idea of preinduction.

Earlier, I described the reaction norm of a trait as the sum of all reactions between trait substrate, inner genes ${ }^{41}$ and outer environmental stimuli. The result of these reactions are the visible forms of the relevant trait, for example high, middle, or short helm-heights and blue, red, or white flower colors.

For a sharper delineation of this idea it would be good to group the associated reactions into categories (for a quantitative character, there will be many such). Of these categories, only two fit the definition of my earlier conception of the reaction norm term.

First category ("Determination"): Reactions between substrate $(S)$ and active genes (genecomplexes). These reactions directly precede the activation of the other genes that have been latent, and therefore the association reaction

\footnotetext{
${ }^{39} \mathrm{MOP}$ note: this refers to the results of De Vries, which were thought to be due to accidental hybridization when re-examined.

${ }^{40}$ MOP note: MacBride's 1932 review in Nature of Woltereck's work states outright that Woltereck proved the inheritance of acquired characters. I believe he refers to the present work.

${ }^{41} \mathrm{MOP}$ note: Anlagen.
} 
between gene $(G)$ and activator $(a)$. We wish to consolidate both reaction processes under the name of determination. This includes all of those visible characters $(R)$ that the substrate may develop via different genes (or geneintensities; see section V.b.).

Reaction formula:

$$
\begin{array}{r}
S+G_{1}+a=R_{1} \\
S+G_{2}+a=R_{2} \\
\text { etc. }
\end{array}
$$

Second category ("Induction"): While the determination of many (qualitative) traits cannot be affected by environmental factors, there are very many (particularly quantitative) reactions that are broadly dependent on the environment. In the simplest case, a constant gene complex $(G)$ is responsible, in which case the reactions therefore proceed between $S, G$, $a$, and the active environmental factor $(M)$, Again, this results in the possible appearances of the trait form.

Reaction formula:

$$
\begin{aligned}
\left(S+G_{1}+a\right)+M_{1}= & R_{1} \\
\left(S+G_{2}+a\right)+M_{2}= & R_{2} \\
& \text { etc. }
\end{aligned}
$$

There appears to be nothing more for preinduction to add beyond these two reaction categories, after we recognize that preinduction is part of the reaction norm. However, closer consideration shows that we must formulate two further categories to cover the extent of the reaction norm. These categories fully correspond in their mutual behavior to those already covered. However, the reactions do not proceed in the trait substrate or body part (as in induction), but rather only in the germ cells.

Third category ("predetermination"): The competing genes of an alternative trait (for example wing scale pigmentation, or sexuality) or of a quantitative trait (helm height) experience an alteration in the germ cells. These

\footnotetext{
${ }^{42}$ Here we leave unstated whether the environment prefers to act through $S, G$, or $a$, or whether the development of inhibiting substances is responsible; I discussed these four possibilities earlier.
}

alterations effect the "maturation" or activation competence of a previously uninfluenceably resting anlage-substance. The activation competence depends on reactions between the genes $(G)$ and some unknown factors $\left(X^{t}\right)$. Regarding these factors, we know only that they are bound to a certain time period (periodic sexuality alteration of Daphnia, page 151 of the original cited work) and that they can cause an inhibition of activation competence that appears only at some specific time.

The result of the reaction is not a visibly developing character of the body substrate but rather an invisible character, unlike the first two categories. This character corresponds to the valence of the gene $\left(G^{v}\right)$. These degrees of valence are known by the names prevalence, dominance, epistasis, recessivity, and hypostasis.

Reaction formula:

$$
\begin{aligned}
G+X^{t_{1}}= & G^{v_{1}} \\
G+X^{t_{2}}= & G^{v_{2}} \\
& \text { etc. }
\end{aligned}
$$

We can understand "valence" as perhaps the greater or lesser affinity for activators. In the sense of our above conceptual model, this is represented by a greater or lesser number of open haptophores.

A similar time-dependent change of valence (the "dominance shifts" of some authors) can also occur in the trait-cells rather than the germcells, such as for the banding of snails (Lang), the pigmentation of axolotl (Häcker), and the pigmentation of mice (Plate).

Fourth category ("preinduction"): Similar to the reactions of category 2 , predetermined processes can be sensitive to external influences. Here we approach preinduction, the topic of this communication. These reactions occur in the germ cells, involving genes $(G)$, the "maturation" factor $\left(X^{t}\right)$, and external conditions $(M)$. The result of the reaction is again an invisible character of the organism, the degree of valence of the genes $\left(G^{v}\right)$. 
Reaction formula:

$$
\begin{aligned}
\left(G+X^{t_{1}}\right)+M_{1}= & G^{v_{1}} \\
\left(G+X^{t_{2}}\right)+M_{2}= & G^{v_{2}} \\
& \text { etc. }
\end{aligned}
$$

In this brief derivation of the four types of reactions, I have neglected all complications arising from the composition of gene-complexes from different factors ("heritable formulae" of the character; one thinks of Matthiola, Mus, Antirrhinum!). Further, for each reaction scheme I present only some variables. It is apparent that the content of the heritable reaction norm is by no means exhausted by these schemes.

It is obvious that, even for traits so apparently simple as helm-height, these gears of reaction may be quite convoluted. Further analyses are likely to find this. However, one point emerges from this complexity: the entire reaction norm is inherited with the genes of a character 43 .

In terms of the theory of descent, the goal of this article was merely an analytical confirmation. Specifically, we learned that typealteration through the influence of the environment (we do not discuss what this means

\footnotetext{
${ }^{43}$ A question, which can be posed here only in shortened form, is the following: In what fashion or in which form can such diverse regularity be inherited? It is difficult enough to imagine how one or more genes can exist in some germ cells for each single character in the body. In particular, when we try to understand these characters (in no very large number) and their genes not as plain molecules but rather only as "scaffolding nuclei" (or as autonomous units of a complex stem scaffold?). Much more difficult is the question of how the various reaction norms can be bound with these scaffold nuclei.

This question cannot be answered, or rather only in the form of solving a very general and not really biological problem (that is to say, not solving the problem). Reaction norms surely belong to the "intensive manifolds", whose meaning for the organic world has been emphasized especially by Driesch [MOP note: Woltereck and Driesch appear to be using a philosophical term from Bergson, "intensive Mannigfaltigkeit", as a name for those entities that cannot be analyzed using deterministic or reductionistic methods]. And surely they belong in the same conceptual category as the "constants", which play a role in biology just as in physics, chemistry, and mineralogy. The chemical affinity constants, the specific heats, the crystallographic and also the formative biological constants are similarly intensive manifolds, just like the reaction constants of variable traits analyzed here.
}

here) sets in only if the reaction norm changes. Preinduction is not type-alteration, because it can be included in the inherited reaction norm.

\section{Discussion}

Professor Hesse (Berlin) suggests that to avoid future confusion regarding cell nuclei, instead of "scaffold nuclei", to use another expression such as "scaffold knots". 